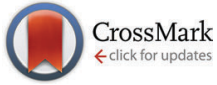

Cite this: New J. Chem., 2015, 39, 5161

Received (in Victoria, Australia) 10th March 2015 Accepted 13th April 2015

DOI: $10.1039 / c 5 n j 00602 c$

www.rsc.org/njc

\title{
New emerging rare-earth free yellow emitting 2D BCNO nanophosphor for white light emitting diodes $\dagger$
}

\author{
Jaya Dwivedi, ${ }^{\text {ab }}$ Pawan Kumar, ${ }^{\text {ab }}$ Garima Kedawat $^{\mathrm{c}}$ and Bipin Kumar Gupta*a
}

\begin{abstract}
We have demonstrated a new emerging rare-earth free highly-efficient two dimensional (2D) boron carbon oxynitride (BCNO) yellow emitting nanophosphor with high quantum efficiency for white light emitting diode (WLED) devices. This BCNO nanophosphor exhibits 2D layered structures analogous to hexagonal BN phase. Further, the EELS and XPS results confirm the nanophosphor consisted of B, C, N and O elements. The BCNO nanophosphor shows a broad highly intense yellow emission band centered at $580 \mathrm{~nm}$ corresponding to $470 \mathrm{~nm}$ excitation wavelength with a quantum efficiency approaching $89 \%$. This novel nanophosphor with strong emission has subsequently been integrated to chip on board (CoB) based blue LEDs in order to fabricate WLEDs devices with a color rendering index of 92. Low color temperature (4899) and better CIE color coordinates $(x=0.3496, y=0.3679)$ of a fabricated WLEDs device supports a 2D BCNO nanophosphor that could be an exceptional choice for CoB based WLEDs. Hence, our method provides a facile synthesis of rare-earth free 2D lightweight BCNO nanophosphor and its integration with COB based blue LEDs for next generation advanced solid state white light applications.
\end{abstract}

\section{Introduction}

Recently, two-dimensional (2D) nanomaterials have been emerging as an important interest for new access of 2D systems because of their outstanding physical, chemical, optical, and structural properties. $^{1,2}$ The dimensionality of a material determines its characteristics to a great extent. The discovery of graphene, an atom thick 2D layered structure of graphite, has stimulated tremendous research on $2 \mathrm{D}$ materials because of their exotic properties in this regime. These materials display noteworthy potential in the arena of electronics devices, optoelectronic devices, van der Waals hetero structures and in various other technological applications. ${ }^{3}$

Intensive studies regarding graphene in recent years marked it as a very intriguing material with astonishing electronic, mechanical and thermal properties, such as high electron mobility and very low resistivity to name a few among others. ${ }^{1,4,5}$ However, even after being such an exquisite material, graphene possesses

\footnotetext{
${ }^{a}$ CSIR - National Physical Laboratory, Dr K S Krishnan Road, New Delhi, 110012, India. E-mail: bipinbhu@yahoo.com; Fax: +91-11-45609310; Tel: +91-11-45609385

${ }^{b}$ Academy of Scientific and Innovative Research (AcSIR), CSIR - National Physical Laboratory, New Delhi, 110012, India

${ }^{c}$ Department of Physics, Kalindi College, University of Delhi, New Delhi, 110008, India

$\dagger$ Electronic supplementary information (ESI) available. See DOI: 10.1039/ c5nj00602c
}

a disadvantage of being sensitive to ambient conditions so much that its properties can be wrecked by materials present in its proximity. For a system to be able to mimic graphene and yet not entertain alterations in its electronic properties by environment, the material particularly must have a large electronic band gap and no dangling bonds. Consequently, hexagonal boron nitride (h-BN) has drawn considerable attraction of investigators due to its isoelectric resemblance with graphene; its alternating nitrogen and boron atoms bear a similar atomic arrangement to carbon in graphene. Despite being structurally similar to graphene, h-BN enjoys differences: being a wide band-gap semiconductor ( $\sim 6 \mathrm{eV}$ ), high corrosion resistance, better thermal, chemical and mechanical stability, intense UV-emission, high thermal stability, and many others. ${ }^{6-8}$ The primary luminescence peak for pure h-BN was measured at $215 \mathrm{~nm}(5.77 \mathrm{eV}) .{ }^{9,10}$ This UV emission is ascribed to the radiative decay of lowest lying exciton, called free exciton because it is independent of any structural defects in a material. However, a red shift was observed due to deep level impurities such as oxygen and carbon. ${ }^{11,12}$ The main peak displays several phonon replicas on the low energy side due to electron-phonon coupling as explained by Han et al. ${ }^{11}$ who showed that the splitting between the peaks, i.e. the vibrational frequency, changes as a function of the boron isotope. Moreover, calculations also indicated the stability of oxygen in substitution for nitrogen atoms in a host BN lattice. The vacancies and other impurities, such as carbon, could give rise to deep levels as well. To be precise, by controlling the content of carbon and oxygen, 
an additional way is provided to tune the optical properties. In addition, theories based on quantum chemical calculations predicted that an atomic-level hybridization of h-BN and graphite produce a new semiconducting compound $\mathrm{BNC}_{x}$ with a tunable band gap energy between that of graphite and h-BN depending on its composition $(x)$ and the atomic configuration in the layer. $^{13-15}$ This has a strong impact on potential applications of these materials in hydrogen storage, ${ }^{12,13}$ electronic devices, ${ }^{14,15}$ electron field-emission, ${ }^{16}$ electrocatalysis,${ }^{17}$ as a supercapacitor, ${ }^{18}$ and metal-free phosphors. ${ }^{19,20}$ Unfortunately, BCN systems are much harder to synthesize and it is difficult to improve the quantum yield of this system, which has only single emission. To date, $\mathrm{BNC}_{x}$ compounds with $x$ varying from 0 to 7 have been prepared by various methods and their photoluminescence (PL) properties have also been investigated. ${ }^{16-21}$

It has been speculated that compounds of a $\mathrm{BNC}_{x}$ series may become an appropriate candidate for solid state lighting materials, especially for white light emitting diodes (WLEDs). Until now, WLEDs heavily relied on rare-earth doped phosphor (YAG:Ce), which are optically pumped by the emission from blue LEDs. ${ }^{22}$ The combined effect of electroluminescence from blue LEDs and photoluminescence of phosphors results in white light. Most of the currently used phosphors in WLEDs are oxides, nitrides, sulfides or oxysulfides doped with rare-earth materials. ${ }^{23-25}$ These rare-earth materials are available in meager quantity in nature and, hence, are very expensive. Also, their synthesis process requires high temperature and pressure, consequently resulting in large power consumption. Besides, these are heavy metals and their chlorides and oxides are toxic and hazardous for human health as well as for the environment. Hence, the use of RE-ions doped phosphors is limited in the future by being economically unfeasible and environmentally harmful. Thus, there is a need to prepare phosphors which are thermally and chemically stable, have non-toxic behaviour, show high luminescence efficiency, and have a broad excitation and emission spectrum besides being inexpensive. Few of the recently developed phosphors like quantum dots of ZnS:Mn, CdS, CdSe, ZnCuInS/ZnS etc. do not contain rare-earths but still, these materials contain toxic elements like sulfur, cadmium and selenium. ${ }^{22}$ Therefore, the development of a novel "yellow" emitting phosphor free from rare-earths as well as toxic metals, which can be synthesized at relatively low temperatures under ambient atmospheric conditions, is desirable for WLEDs applications. A very recent report [I. Akasaki, H.; et al., DOI: $10.1038 /$ nphys3147; Nobel Prize awarded on 7th October 2014] describing the invention of efficient blue light-emitting diodes, which enabled bright and energy-saving white light sources, could potentially reignite searches for WLEDs applications in a broad range of layered rare-earth free materials.

In light of the above requirements, the present work explores a new prospect for developing a rare-earth free 2D lightweight boron carbon oxynitride (BCNO) nanophosphor for WLEDs. In this series, the yellow emitting 2D BCNO nanophosphor shows a promising future in this direction that satisfies all the desirable properties. A few attempts on the synthesis of this material have been explored in detail by several research groups and all of them showed PL properties of BCNO nanocrystals phosphor. ${ }^{26-29}$
However, we attempted the new emerging 2D based BCNO nanophosphor after a slight modification of the reported synthesis method. ${ }^{30,31}$ The incorporation of oxygen to the BCN system has drastically enhanced its color tuning range, which extends from violet to red regions of photoluminescence spectra. The theoretical calculations ${ }^{21,30}$ and experimental evidence ${ }^{19,31-36}$ have indicated that tunable visible emissions with high quantum yields can be achieved using BCNO compounds which have metal-free phosphors with low toxicity properties. In addition, high external quantum efficiency and a broad range of excitation wavelengths also were obtained. Until very recently, few works have been reported on the synthesis of BCNO phosphors using thermal catalytic CVD, solid-state reactions, microwave plasma CVD, and a one-step liquid process with applications in WLEDs. $^{36,37}$

Concisely, in this investigation, we have presented a comprehensive approach towards synthesis of a 2D layered BCNO nanophosphor by a facile and low temperature method comparative to known rare-earth based bulk material synthesis. ${ }^{3}$ Raw materials used in the synthesis process are readily available and inexpensive such as boric acid, polyethylene glycol (PEG), and urea/ carbamide, which makes the overall synthesis very economical. Moreover, the shape dependent structural/microstructural studies, as well as their photoluminescence properties, of the synthesized nanophosphor were explored in detail. Finally, we successfully integrated the as-synthesized nanophosphor with commercial CoB (chip on board) based blue LEDs for fabrication of WLEDs.

\section{Experimental}

\subsection{Synthesis of 2D BCNO nanophosphor}

The 2D BCNO nanophosphor was prepared by a facile, inexpensive, and customized auto-combustion method. The synthesis process was focused on improving uniform emission with high quantum efficiency. Initially, $0.10 \mathrm{~mol}$ of boric acid (boron source, $\mathrm{H}_{3} \mathrm{BO}_{3}$ ) and $1.00 \mathrm{~mol}$ of urea or carbamide (nitrogen source, $\mathrm{CO}$ $\left(\mathrm{NH}_{2}\right)_{2}$ ) were dissolved in $120 \mathrm{~g}$ of ultrapure water. Subsequently, the dispersing agent, polyethylene glycol (PEG, $M_{\mathrm{w}}-10000$, and $\left.\mathrm{H}\left(\mathrm{OCH}_{2} \mathrm{CH}_{2}\right)_{n} \mathrm{OH}\right)$ was added to the prepared solution and stirred vigorously at $100{ }^{\circ} \mathrm{C}$ until a clear transparent aqueous solution was obtained. The chemical structure of boric acid, polyethylene glycol and carbamide are shown in Fig. S1 (see ESI $\dagger$ ). The XRD patterns of PEG, carbamide and boric acid are given in Fig. S2 (see ESI $\dagger$ ), and are indexed by JCPDS Card No. 45-0706, 86-2276 and 78-0459, respectively. These clearly demonstrate the presence of their respective pure phases. The resulting solution was heated in closed box type furnace to $400{ }^{\circ} \mathrm{C}$ at a rate of $6{ }^{\circ} \mathrm{C} \mathrm{min}{ }^{-1}$ and kept at this temperature for $10 \mathrm{~min}$ to form pyrolysate (fluffy dry-gel powder form). The closed box furnace was used to control oxygen during the synthesis process. The pyrolysate step is an important one in which an organic compound is pyrolysed under conditions that do not allow organic compounds to vanish by combustion, so that the $\mathrm{C} / \mathrm{N}$ ratio remains constant. The choice of raw material is also quite important for the synthesis of BCNO nanophosphors. 
The suitability of raw materials was decided on the basis of higher photoluminescence intensity of BCNO nanophosphors. We made several statistical runs with different carbon sources such as EG, TEG, PEG 100, PEG 200 and PEG 10000 to synthesize 2D BCNO nanophosphors for better PL intensity. The PL spectra of various carbon sources based BCNO nanophosphors are shown in Fig. S3 (see ESI $\dagger$ ). The best result was obtained using PEG with a molecular weight of 10000 . Finally, we only focused on PEG as an efficient carbon source to synthesize 2D BCNO nanophosphors. The resulting pyrolysate mixture turned into a grey solid due to carbonization of a part of the raw material causing a pyrolysis reaction involving deoxidation and dehydrogenation. The pulverized pyrolysate mixture was disintegrated in order to have a uniform carbon composition. Then, the mixture was transferred to a ceramic crucible and heated to $800{ }^{\circ} \mathrm{C}$ in a box furnace at the rate of $25{ }^{\circ} \mathrm{C} \mathrm{min}^{-1}$ for $10 \mathrm{~min}$ to obtain the BCNO precursor. The as-prepared precursor was subsequently annealed at $750{ }^{\circ} \mathrm{C}$ for 45 min under the ambient conditions to obtain homogeneity throughout the mass. Consequently, a yellow bulk mass was obtained with an $86 \%$ yield. The schematic diagram to synthesize 2D BCNO nanophosphor and its proposed chemical structure with repeated unit cell is shown in Fig. 1. The protocol for the synthesis of 2D BCNO nanophosphors using an auto-combustion method is given in Scheme $\mathrm{S} 1$ (see $\mathrm{ESI} \dagger$ ). In this process the $\mathrm{B} / \mathrm{N}$ ratio was kept constant, whereas the $\mathrm{B} / \mathrm{C}$ ratio was varied in order to obtain desired wavelengths, as shown in Table S1 (see ESI $\dagger$ ). It is quite evident that the emission color wavelength can be easily tuned from violet to red regions simply by varying the $\mathrm{B} / \mathrm{C}$ ratio. As we required a yellow nanophosphor for proposed application. Therefore, here we have only described the synthesis, characterizations, and properties of the $2 \mathrm{D}$ BCNO yellow nanophosphor.

\subsection{Characterization}

The phase purity identification, as well as gross structural characterization, was performed by XRD (Rigaku: MiniFlex, $\mathrm{CuK} \alpha_{1}$; $\lambda=1.5406 \AA$ ), which utilizes the principle of Bragg Brantano Geometry. The X-ray scan range went from $10^{\circ}$ to $80^{\circ}$ at a scanning rate of $2^{\circ}$ per minute. Prior to the XRD measurement, the diffractometer was calibrated using silicon powder as the reference material $\left(d_{111}=3.1353 \AA\right) .{ }^{38}$ Accurate lattice parameters were also obtained through a least square fitting method using computer-based unit cell refinement software. ${ }^{39}$ The chemical bonding state was examined with Fourier transformation infrared (FTIR) spectroscopy with a NICOLET 6700 instrument using $\mathrm{KBr}$ pellets. The spectrum was collected at a resolution of $2 \mathrm{~cm}^{-1}$ and each spectrum used an average of 32 scans. The X-ray photoelectron spectroscopy (XPS) analysis was performed in an ultrahigh vacuum (UHV) chamber equipped with a hemispherical electron energy analyzer (PerkinElmer, PHI1257) using a nonmonochromatized $\mathrm{Al} \mathrm{K} \alpha$ source (excitation energy of $1486.7 \mathrm{eV}$ ) with a base pressure of $4 \times 10^{-10}$ Torr at room temperature. The thickness of 2D layered BCNO nanophosphors was measured by atomic force microscopy (AFM, model: NT-MDT Solver Scanning probe Microscope). Raman spectra were obtained using Renishaw InVia Raman spectrometer, UK with an excitation source of $514.5 \mathrm{~nm}$. The surface morphology and microstructural characterizations were carried out by scanning electron microscopy (SEM, Model No. EVO MA 10 VPSEM) and high resolution transmission electron microscopy (HRTEM, Model No. Technai G20-twin, $200 \mathrm{kV}$ with super twin lenses having point and line resolution of $0.144 \mathrm{~nm}$ and $0.232 \mathrm{~nm}$, respectively) equipped with energy dispersive X-ray analysis (EDAX) facilities for elemental studies. The chemical composition of the 2D BCNO nanophosphor was analyzed by an electron energy-loss spectrometer (EELS) using a spectrometer attached to the transmission electron microscope. UV-visible spectra were collected using a high resolution UV-Vis spectrophotometer (MODEL No. LS 55). An electron paramagnetic resonance (EPR, Bruker A300 EPR) spectrum was collected to give a preliminary explanation of the origin of the fluorescence. The photoluminescence (PL)

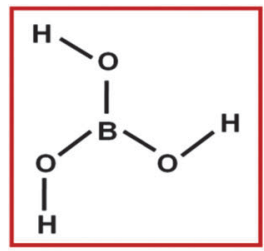

Boric Acid

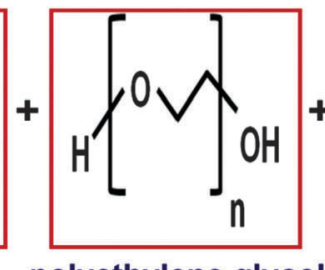

polyethylene glycol

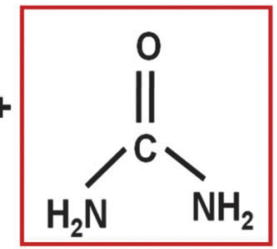

Carbamide

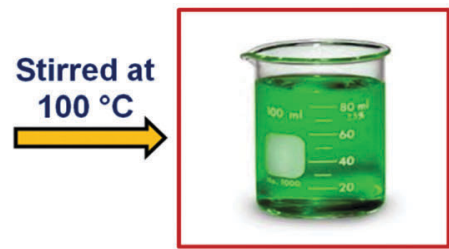

Mixed aqueous solution

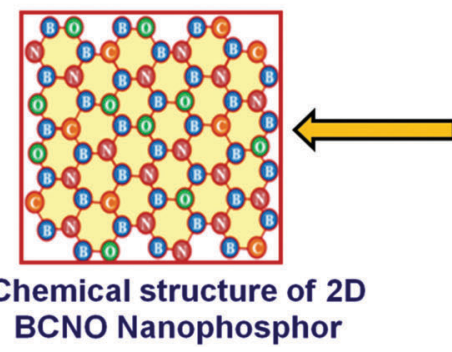

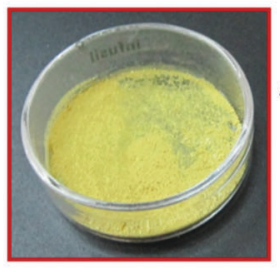

2D BCNO Nanophosphor

\section{Annealing at}
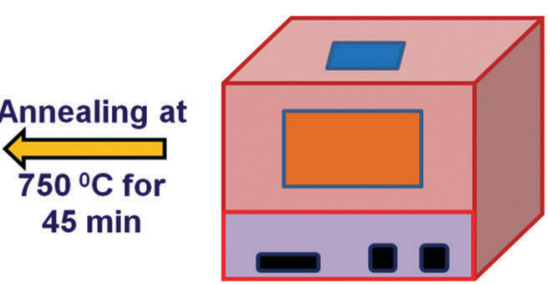

Heating at $800^{\circ} \mathrm{C}$ for $10 \mathrm{~min}$
$45 \mathrm{~min}$

Fig. 1 Schematic diagram to synthesize 2D BCNO nanophosphor and its proposed chemical structure with repeated unit cell. 
spectra of nanophosphors were carried out using a photoluminescence spectrometer (Edinburgh, FLSP-920), where a Xenon flash lamp acts as source of excitation. Time-resolved spectroscopy was performed using a photoluminescence spectrometer with an EPL $375 \mathrm{~nm}$ picoseconds pulsed diode laser as a source of excitation. In order to estimate the absolute quantum efficiency, we used an integrating sphere, which was equipped with an Edinburgh spectrometer F-900 instrument. The estimation of quantum yield is based on the integrated fraction of luminous flux and radiant flux as per an established standard method. ${ }^{40}$ PL mapping was performed with a WITech alpha 300R+ Confocal PL microscope system (WITech GmbH, Ulm, Germany), where a $375 \mathrm{~nm}$ diode laser was used as a source of excitation. Color temperature and CIE color coordinates were measured using a colorimeter C1210, serial no. 1296104, and luminance of nanophosphors-based WLEDs was calculated by a luminance meter series L1000. All the optical photographs were taken from a red sensitive digital CANON PowerShot SX600 HS (16× Megapixels, $5 \times$ optical zoom) camera.

\section{Results and discussion}

\subsection{Structural analysis}

To examine the phase purity, we performed the XRD of as-synthesized 2D BCNO nanophosphor. The XRD pattern is shown in Fig. 2a which indicate the existence of $\mathrm{a}_{2} \mathrm{O}_{3}, \mathrm{BN}$ and graphitic carbon lattice. It exhibits two broad peaks centered at $26.6^{\circ}$ and $41.5^{\circ}$ which signifies the formation of hexagonal boron nitride (h-BN; JCPDS no. 74-1978). ${ }^{41}$ Besides, there are three more sharp and distinct peaks at $14.5^{\circ}, 27.7^{\circ}$ and $42.8^{\circ}$ corresponding to the crystal planes of cubic $\mathrm{B}_{2} \mathrm{O}_{3}$ (JCPDS no. 06-0297). These extraneous peaks are a consequence of un-reacted boric acid. The graphitic carbon peak ${ }^{42}$ (JCPDS no. 75-1681) at $26.2^{\circ}$ appears together with a h-BN peak at $26.7^{\circ}$. These peaks are de-convolved by Gaussian fitting, as shown in Fig. 2b; the black square mark in Fig. 2a shows the place where the fitting was performed. It clearly demonstrates the presence of graphitic carbon, h-BN and $\mathrm{B}_{2} \mathrm{O}_{3}$. The graphene-facilitated formation of h-BN was observed and these overlapped signals indicate the hexagonal phase of the 2D BCNO nanophosphor. The inset of Fig. 2b depicts the proposed unit cell of 2D BCNO layers. Each boron atom is surrounded by three nitrogen atoms from which one of the nitrogen atoms has been replaced by a carbon atom and another one by an oxygen atom to form a BCNO unit cell. These carbon and oxygen atoms behave as impurity atoms in a hexagonal-boron nitride (h-BN) lattice.

A typical FTIR spectrum of as-synthesized 2D BCNO nanophosphor in range of $600-2000 \mathrm{~cm}^{-1}$ is shown in Fig. 2c. It is clear that the 2D nanosheet structure arises in the as-synthesized BCNO nanophosphor. The strong absorption bands having a centre at $\sim 770 \mathrm{~cm}^{-1}$ and $\sim 1420 \mathrm{~cm}^{-1}$ unambiguously denote the presence of $\mathrm{B}-\mathrm{N}$ linkage (stretching mode), which can be attributed to the in-plane and out-of-plane vibration modes of the $\mathrm{sp}^{2}$ hybridized $\mathrm{BN}{ }^{43,44}$ The broadening observed in the $\mathrm{B}-\mathrm{N}$ absorption bands can be ascribed to the incorporation of carbon atoms into the host $\mathrm{BN}$ lattice. ${ }^{45}$ A sharp edge related to the $\mathrm{B}-\mathrm{C}$ stretching bond is observed at $\sim 1240 \mathrm{~cm}^{-1}$. ${ }^{46}$ The two distinctly separate bands observed at $\sim 930 \mathrm{~cm}^{-1}$ and $\sim 1070 \mathrm{~cm}^{-1}$ correspond to $\mathrm{B}-\mathrm{N}-\mathrm{B}$ bonds and $\mathrm{N}-\mathrm{B}-\mathrm{O}$ bonds, respectively. ${ }^{47}$ The vibrational band peaking at $\sim 680 \mathrm{~cm}^{-1}$ is attributed to the $\mathrm{B}-\mathrm{O}$ bonds, which are presented in the BCNO nanostructure. Further, the quality of the 2D BCNO layer was confirmed through the AFM technique. Fig. 2d shows a typical AFM image of the as-synthesized 2D BCNO nanophosphor, which exhibits a sheet-like nanostructure establishing its 2D structure. The region marked by blue arrows in Fig. S4a (see ESI $\dagger$ ) shows the place where a line-scan profile was taken to calculate the roughness and thickness of layers. The corresponding line-scan profile indicates a thickness of $\sim 2 \mathrm{~nm}$ corresponding to a few h-BN layers, which can be clearly seen in Fig. S4b (see ESI $\dagger$ ). To ascertain the crystallinity, as well as vibration modes of the $2 \mathrm{D}$ BCNO nanophosphor, we performed Raman spectroscopy, as shown in Fig. S5 (see ESI $\dagger$ ). It clearly demonstrates the strong peak and oxide phase of the BCNO nanophosphor.

Further, the survey-scanned XPS spectrum of the as-synthesized 2D BCNO nanophosphor is given in Fig. 2e. Prior to the XPS measurement, calibration of the binding energies was done by keeping the C1s peak at $284 \mathrm{eV}$, as a standard. The other significant peaks present at $190.27 \mathrm{eV}, 397.50 \mathrm{eV}$ and $531.80 \mathrm{eV}$ correspond to the $\mathrm{B} 1 \mathrm{~s}, \mathrm{~N} 1 \mathrm{~s}$ and $\mathrm{O} 1 \mathrm{~s}$, respectively. The XPS results ensure the formation of BCNO nanocomposite. The XPS core level spectra of $\mathrm{B} 1 \mathrm{~s}, \mathrm{C} 1 \mathrm{~s}, \mathrm{~N} 1 \mathrm{~s}, \mathrm{O} 1 \mathrm{~s}, \mathrm{O} 2 \mathrm{~s}$ and OKLL regions for the 2D BCNO nanophosphor are shown in Fig. S6 (see $\mathrm{ESI} \dagger$ ). A detailed study on the bonding structure of the 2D BCNO nanophosphor was made using XPS B1s, C1s and N1s profiles, which are deconvoluted by Gaussian fitting to get information about bonding characteristics, as shown in Fig. $2 \mathrm{f}-\mathrm{h}$. The highresolution B1s spectrum of the sample (Fig. 2f) shows three peaks at 190.50, 191.69 and $192.91 \mathrm{eV}$, which are ascribed to $\mathrm{B}-\mathrm{O}, \mathrm{B}-\mathrm{N}$, and $\mathrm{B}-\mathrm{C}$ bonds, respectively. The high-resolution C1s spectrum (Fig. 2g) shows four peaks at 283.01, 284.04, 285.43 , and $287.05 \mathrm{eV}$, which are attributed to $\mathrm{C}-\mathrm{B}, \mathrm{C}-\mathrm{C}, \mathrm{C}=\mathrm{C}$, and $\mathrm{C}-\mathrm{O}$ bonds, respectively. The high-resolution N1s spectrum (Fig. 2h) reveals three peaks at 397.44, 398.98, and $401.04 \mathrm{eV}$, which are attributed to the pyridinic $\mathrm{N}, \mathrm{C}-\mathrm{B}-\mathrm{N}$ and graphitic $\mathrm{N}$ bonds, respectively. The above results indicate that the $\mathrm{B}-\mathrm{N}$, $\mathrm{B}-\mathrm{C}, \mathrm{B}-\mathrm{O}$ and $\mathrm{C}-\mathrm{O}$ bondings are formed in the synthesized 2D BCNO nanophosphor. The basic structure of the sample is the heterocyclic compound unit. The hexagonal phase is also supported by the FTIR spectrum.

\subsection{Surface morphology and microstructural analysis}

To explore the surface morphology and microstructure of the 2D BCNO nanophosphor, we performed SEM and TEM/HRTEM measurements. Prior to SEM and TEM/HRTEM analysis, the BCNO powder samples were sonicated in ethanol using ultrasonicator operating at $25 \mathrm{kHz}$ frequency to avoid any agglomerations in the sample, consequently leading to better SEM and TEM/HRTEM image quality. Fig. 3a represents the SEM image of the as-synthesized 2D BCNO nanophosphor. It shows a thin, 2D randomly oriented layered nanostructure of the 

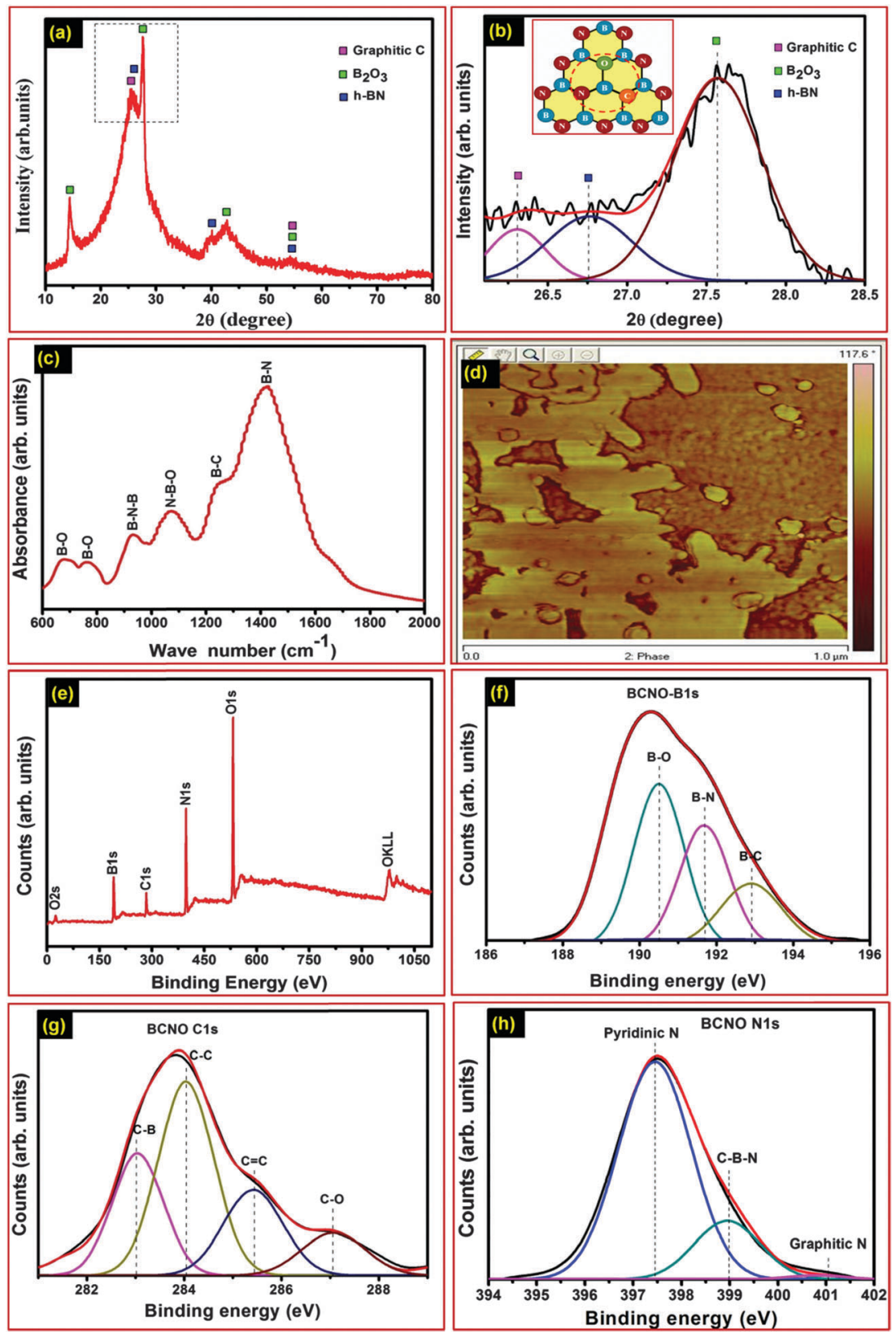

Fig. 2 (a) XRD of the 2D BCNO nanophosphor. (b) Deconvoluted spectra signify the formation of hexagonal boron which was performed from the rectangle marked region in (a); it clearly demonstrates the presence of graphitic carbon, $\mathrm{h}-\mathrm{BN}$ and $\mathrm{B}_{2} \mathrm{O}_{3}$ and the inset exhibits the proposed unit cell of $\mathrm{BCNO}$ layered structure. (c) FTIR spectrum of the $2 \mathrm{D} B C N O$ nanophosphor in a range of $600-2000 \mathrm{~cm}^{-1}$. (d) AFM micrograph of the $2 \mathrm{D} B C N O$ nanophosphor. (e) Survey-scanned XPS spectrum of 2D BCNO nanophosphor and XPS profile of (f) B1s, (g) C1s and (h) N1s; these curves are deconvoluted by Gaussian fitting, thereby indicating possible bonding structures.

nanophosphor of irregular shape with an average size of the layered sheet $\sim 2 \mu \mathrm{m}$; this is distinctly visible in the magnified view, as shown in inset of Fig. 3a. The TEM image of the 2D BCNO nanophosphor is shown in Fig. 3b. It signifies that various flakes have combined together to form a 2D layered nanostructure, as shown by the yellow marked arrows. A magnified view of the TEM image is given in the inset of Fig. 3b. It also candidly asserts its layered structure with thickness in a range of $2 \mathrm{~nm}$. A typical HRTEM image of the specified area marked by a red circle in the inset of Fig. 3b is shown in Fig. 3c. The precise observation of the HRTEM image indicates the various distinct lattice fringes with different lattice spacing. This signifies that the BCNO nanophosphor is layered in nature and it is composed of few layers. The lattice spacing of the constituent-layered nanostructure is $0.34 \mathrm{~nm}$ corresponding to the (002) planes of h-BN. The 2D BCNO nanophosphor can also be verified by the selected area electron diffraction (SAED) pattern as shown in Fig. 3d. The diffraction rings, although somewhat weak due to the stacking of a few layers, can be distinctly assigned to the (002), (100), (004), and (110) planes of h-BN (JCPDS no. 73-2095). Fig. $3 \mathrm{e}$ is the EDAX 

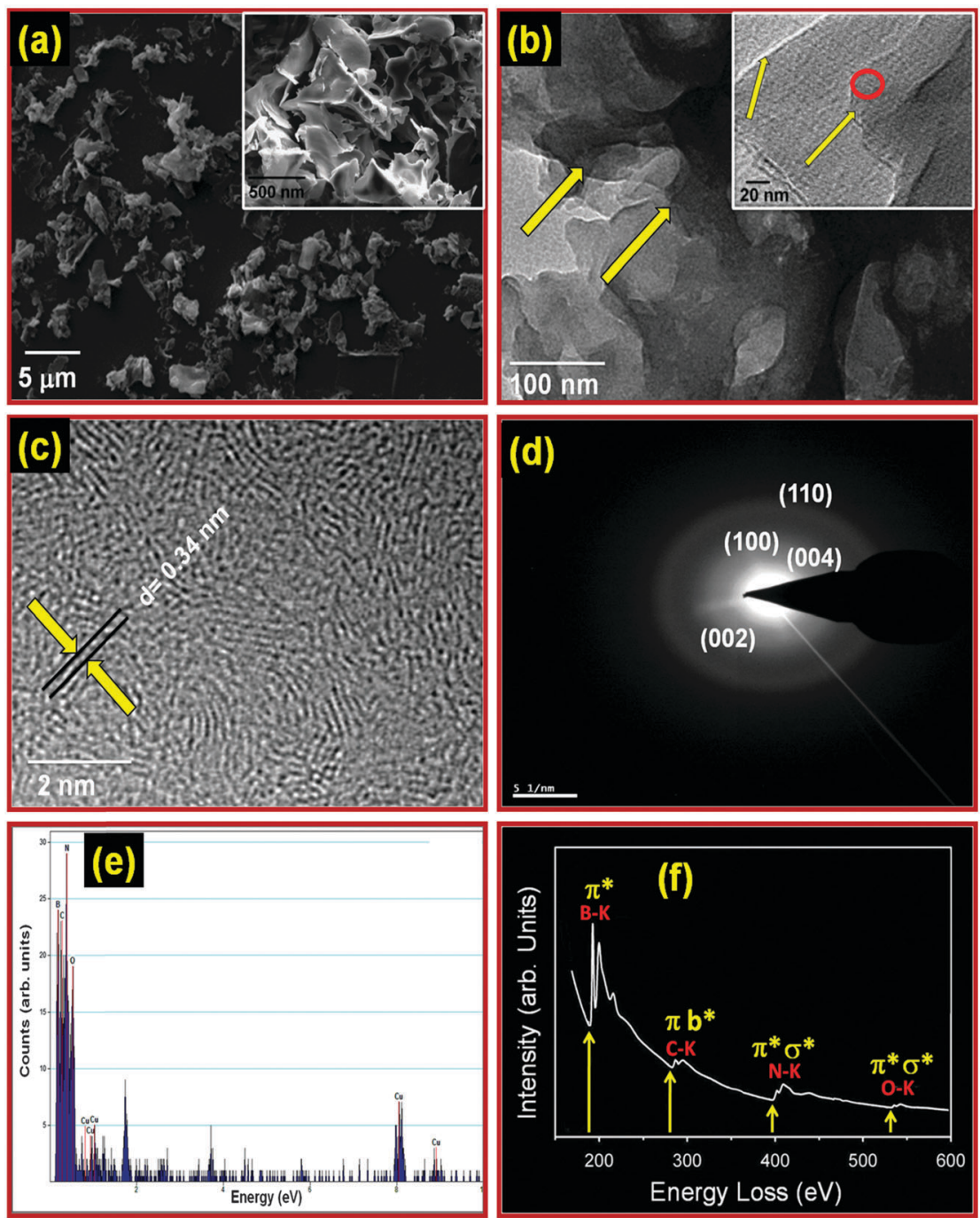

Fig. 3 (a) SEM image of the 2D BCNO nanophosphor; inset shows its magnified view clearly demonstrating the 2D layered structure. (b) TEM image of the 2D BCNO nanophosphor; inset shows magnified view of area marked by yellow arrow in (b). (c) HRTEM image of the 2D BCNO nanophosphor; the image was taken at the specific area marked by the red circle in the inset of (b). (d) SAED of the 2D BCNO nanophosphor. (e) EDAX spectrum of the 2D BCNO phosphor. (f) EELS spectrum of 2D BCNO nanophosphor.

spectrum of the 2D BCNO nanophosphor, the area marked by the red circle in Fig. $3 \mathrm{~b}$ shows the place where the EDAX spectrum was taken. The elements detected by EDAX are largely comprised of $\mathrm{B}, \mathrm{C}, \mathrm{N}$, and $\mathrm{O}$ with mass fractions of $0.13,0.75$, 0.07 , and 0.04 , respectively. This result was consistent with the composition of the precursor used in the experiment. The chemical composition of the BCNO nanophosphor was further analyzed using an electron energy-loss spectrometer (EELS), which measures the energy imparted to a thin $(\sim 200 \mathrm{~nm})$ specimen by fast $(\sim 100 \mathrm{keV})$ incident electrons, from which high spatial resolution of chemical information can be gained. The EELS spectrum is shown in Fig. 3f. It exhibits two sharp peaks of B-K ionization at 193 and $201 \mathrm{eV}$ for $\pi^{*}$ and $\sigma^{*}$ electrons, respectively. As Garvie et al. pointed out, in the case of a BCNO compound, if the B-K ionization for $\pi^{*}$ electrons has three peaks of 189.3, 192.1 , and $194.1 \mathrm{eV}$, the $\mathrm{B}$ atom is surrounded by three elements, i.e., $\mathrm{C}, \mathrm{N}$, and $\mathrm{O}$, respectively. Since the $\mathrm{B}-\mathrm{K}$ ionization for $\pi^{*}$ electrons had only one peak between 192 and $194 \mathrm{eV}$, this meant that the $\mathrm{B}$ atom was intermediately surrounded by $\mathrm{N}$ and $\mathrm{O}$ atoms, which also implied that there were no clear $\mathrm{B}-\mathrm{C}$ chemical bonds in the BCNO compound. The peak at $285 \mathrm{eV}$ is assigned to $\pi^{*}$ bands, while those at 288 and $295 \mathrm{eV}$ are classified as $\sigma^{*}$ bands, in which the peak at $288 \mathrm{eV}$ has $\mathrm{C}-\mathrm{H}^{*}$ features. Previous research showed that materials with weak $\mathrm{sp}^{2}$ hybrid orbitals, such as amorphous carbon, graphite, and solid benzene, can have a $\pi^{*}$ feature; while those having stronger $\mathrm{sp}^{3}$ hybridization, ${ }^{13}$ such as a diamond and solid cyclohexane, exhibit only $\sigma^{*}$ (including $\mathrm{C}-\mathrm{H}^{*}$ ) features with no $\pi^{*}$ bands. The $\mathrm{C}-\mathrm{H}^{*}$ feature can usually be found in organic materials, such as solid benzene and cyclohexane. In the present study, the $\mathrm{C}-\mathrm{H}$ bonds are supposed to come from the organic residue of decomposed PEG. Since these data are evidence that the $\mathrm{C}$ atoms in the BCNO nanophosphor has $\pi$ and $\sigma$ bonds, they should have $\mathrm{sp}^{2}$ hybridization. The $\mathrm{sp}^{2}$ hybrids lie in a plane 
and are oriented toward the corners of an equilateral triangle at angles of $120^{\circ}$ to one another and have a framework consisting mainly of a 6-membered carbon ring. Previously reported EELS data of $\mathrm{C}-\mathrm{K}$ edges from different carbon sources supported the above hypothesis that the carbon in the BCNO compound has an amorphous structure with traces of $\mathrm{C}-\mathrm{H}$ groups (organic residue). These should not have a $\mathrm{sp}^{3}$ structure that is rigid and hard, which can be obtained only under extreme conditions. In the present investigations, the BCNO compounds were prepared at low temperature (below $900{ }^{\circ} \mathrm{C}$ ) and under ambient atmospheric conditions, resulting in a powder with a soft framework ( $\mathrm{sp}^{2}$ hybridization). The ionization peak of $\mathrm{N}$ for the $\pi^{*}$ character is at $403.5 \mathrm{eV}$ and the $\sigma^{*}$ character at $410.5 \mathrm{eV}$. Since the $\sigma^{*}$ peak intensity of $\mathrm{N}$ is higher than that of the $\pi^{*}$ peak, this means the major $\mathrm{N}$ atoms have $\sigma^{*}$ bonds with other elements. The ionization peak spectrum of $\mathrm{O}$ is also shown in Fig. 3f, where the peaks at 537 and $545 \mathrm{eV}$ are for the $\pi^{*}$ and $\sigma^{*}$ character, respectively. Basically, the BCNO phosphor is covalently bonded (with $\pi^{*}$ and $\sigma^{*}$ bonds) to the $\mathrm{B}$ and $\mathrm{O}$ atoms with a soft ( $\mathrm{sp}^{2}$ hybridization) carbon lattice. The structure was proposed on the basis of the deconvolution core level XPS spectra of B1s and C1s, where clearly $\mathrm{B}-\mathrm{C}, \mathrm{B}-\mathrm{O}, \mathrm{B}-\mathrm{N}$ bonds and $\mathrm{C}-\mathrm{B}, \mathrm{C}-\mathrm{C}, \mathrm{C}=\mathrm{C}$ and $\mathrm{C}-\mathrm{O}$ bonds are present in the proposed structure (inset of Fig. 2b) which is also legitimized by EELS results. The obtained EELS data have good consistency with XRD and FTIR results.

In order to understand the presence of delocalized $\pi$ electrons on the surface of BCNO and carbon contained impurity and their important role in luminescence process, we performed electron paramagnetic resonance (EPR) studies. The EPR spectrum was recorded at a frequency $9.866 \mathrm{GHz}$ under room temperature. The EPR spectrum of the 2D BCNO nanophosphor is shown in Fig. S7 (see ESI $\dagger$ ). It reveals that the origin of the EPR signal for h-BN can be assigned to paramagnetic centers of electrons trapped in the nitrogen vacancies of two different types: a three boron center and one boron center. Furthermore, Zunger and Katzir, Anderi et al., and Adobe et al. clarified, based on experimental results as well as quantum chemical calculations, that there exists a close relationship between the formation of a paramagnetic center (nitrogen vacancy) and impurity of carbon, which produces luminescence centers with an energy level of $\sim 4.1 \mathrm{eV}$. In the present BCNO investigation, we can interpret it as a carbon doped hexagonal compound of BNO. The origin of the emission band is also assumed to be related to the impurity level of carbon. The $g$-factor is estimated to be $\sim 2.00019 \pm 0.00004$.

\subsection{Optical spectroscopy analysis}

The UV-Vis absorption spectrum of 2D BCNO nanophosphor is shown in Fig. S8 (see ESI $\dagger$ ). The sample was dispersed in DI water and sonicated for 2 hours before taking the spectrum. The absorption spectrum reveals narrow and broad peaks at $\sim 231(\sim 5.4 \mathrm{eV})$ and $\sim 293(\sim 4.2 \mathrm{eV}) \mathrm{nm}$, respectively. The narrow absorption peak is probably induced by the absorption of valance band electrons, while the wide absorption peak might originate from the absorption of carbon related defects. The observed absorption spectrum data is in good correlation with observed photoluminescence (PL) results, which is further discussed in the next section.
The main aim of the present work is related to fabrication of yellow nanophosphor based white light CoBs. We tailored the $\mathrm{PL}$ emission of the 2D BCNO nanophosphor via fixing the $\mathrm{B} / \mathrm{N}$ ratio and tailoring the $\mathrm{B} / \mathrm{C}$ ratio. The various concentrations of $\mathrm{B} / \mathrm{C}$ ranging from $25 / 0$ to $25 / 0.3(A=25 / 0, B=25 / 0.05, C=25 / 0.10$, $D=25 / 0.15, E=25 / 0.20, F=25 / 0.25$ and $G=25 / 0.3)$ are varied accordingly, subsequently leading to a different color emitting BCNO nanophosphor as shown in Fig. S9 (see ESI $\dagger$ ) and summarized data are given in Table S1 (see ESI $\dagger$ ). The role of the carbon atoms in the 2D BCNO nanophosphor plays a key role in the tailoring of band gap, which accompanies the changes in color emission in the BCNO nanophosphor. ${ }^{36}$ The optical photograph of the 2D BCNO nanophosphor in powder form under room light and same phosphors under $340 \mathrm{~nm}$ (portable UV lamp) excitation wavelength are shown in Fig. S10a and b (see $\mathrm{ESI} \dagger$ ), respectively. Therefore, the suitable values of time, concentration, ratio of $\mathrm{B} / \mathrm{C}$ and temperature are found to be optimum values for the synthesis of the yellow 2D BCNO nanophosphor for WLEDs applications.

The PLE spectrum of the 2D BCNO nanophosphor under $580 \mathrm{~nm}$ emission wavelength is shown in Fig. 4a. We also performed the photo-beaching experiment of the 2D BCNO nanophosphor. The obtained result reveals that the nanophosphor exhibits better stability of PL as shown in Fig. S11 (see ESI $\dagger$ ).

Fig. $4 \mathrm{~b}$ represents the emission spectra of the yellow emitting 2D BCNO nanophosphor at $470 \mathrm{~nm}$ excitation wavelength. The probable two PL mechanism proposed until now on the BCNO nanophosphor are as follows: (i) one possible mechanism is that the emission of the BCNO nanophosphor may be introduced by the core shell $\mathrm{BO}^{-}$and $\mathrm{BO}_{2}{ }^{-}$anions acting as luminescent
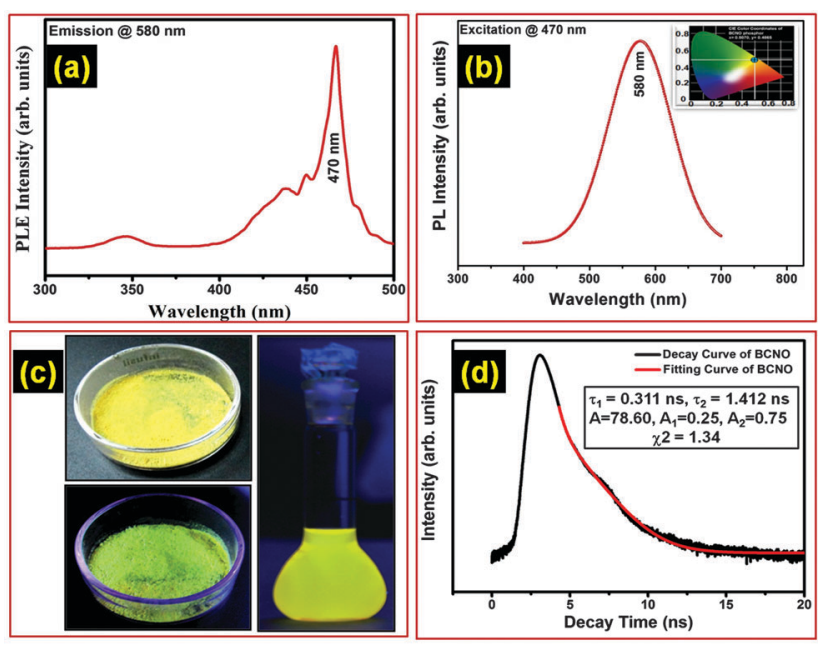

Fig. 4 (a) Excitation spectrum of the 2D BCNO nanophosphor at $580 \mathrm{~nm}$ emission wavelength. (b) Emission spectrum of the 2D BCNO nanophosphor at $470 \mathrm{~nm}$ excitation; inset shows the $\mathrm{CIE}$ coordinates from emission spectra of the 2D BCNO nanophosphor. (c) Top figure exhibits the powder under normal light with yellow body color, while the bottom powder exhibits yellow color under a $340 \mathrm{~nm}$ UV lamp and the right side bottle shows strong yellow color emission of dispersed BCNO powder in DI water when exposed to a $340 \mathrm{~nm}$ UV lamp. (d) Time-resolved photoluminescence decay spectra with a fitting curve and their corresponding parameters of the $2 \mathrm{D} B C N O$ nanophosphor. 
centers ${ }^{35}\left({ }^{2} \pi_{\mathrm{g}} \rightarrow{ }^{1} \sum_{\mathrm{g}}^{+}\right)$and (ii) the other possible mechanism can be attributed to impurity defects, especially nitrogen vacancies in the BCNO nanophosphor. The CIE color co-ordinates (chromaticity diagram) obtained from emission spectra are $x=0.5070$ and $y=0.4865$, as shown in the inset of Fig. $4 \mathrm{~b}$ and located typically in the yellow region. Fig. $4 \mathrm{c}$ represents the synthesized 2D BCNO nanophosphor powder; the top one exhibits the powder under room light with yellow body color, while the bottom powder shows yellow emission under a $340 \mathrm{~nm}$ UV lamp and in the right side a round flask shows strong yellow color emission of dispersed BCNO powder in DI water, when exposed to $340 \mathrm{~nm}$ UV lamp.

On the basis of obtained PL results and based on existing literature report, ${ }^{48}$ a simple proposed energy level diagram is tentatively constructed to explain the spectral properties of the 2D BCNO nanophosphor, as shown in Fig. S12 (see ESI $\dagger$ ). The nitrogen vacancy levels impurity is known as three boron center $\left(\mathrm{v}_{\mathrm{N} 3}\right)$ and one boron center $\left(\mathrm{v}_{\mathrm{N} 1}\right)$ which appears below conduction band 1.0 and $0.7 \mathrm{eV}$, respectively, ${ }^{48}$ Additionally, oxygen impurity may be initiating to produce an energy level of $\sim 4.3 \mathrm{eV}$ below conduction band. The carbon impurity levels are $4.2 \mathrm{eV}$ below the conduction band, while the nitrogen vacancy levels are 0.7-1.0 eV below the conduction band. The excitation at $340 \mathrm{~nm}$ $(3.6 \mathrm{eV})$ is probably induced by the transition from the valence band to the nitrogen vacancy level and the excitation at $470 \mathrm{~nm}$ $(2.6 \mathrm{eV})$ is likely due to the transition from carbon impurity levels to nitrogen vacancy levels. The other long wavelength excitation peaks probably originate from carbon related defects to nitrogen vacancy levels; the carbon related defects include substitutional $\mathrm{CB}$ and $\mathrm{CN}$ defects (whose levels are about $2.2 \mathrm{eV}$ below nitrogen vacancy levels) and interstitial carbon (whose levels are about $1.7 \mathrm{eV}$ below nitrogen vacancy levels). ${ }^{47,49}$ These have been reported to generate deep levels below the conduction band in the range of 2-4 eV. The estimated external quantum efficiency (EQE) of the 2D yellow emitting BCNO nanophosphor is as high as $89 \%$, compared to some rare earth YAG:Ce for WLEDs applications. $^{22}$ Time-resolved photoluminescence (TRPL) was recorded using a time-correlated single photon counting technique, using a $375 \mathrm{~nm}$ EPL pulse diode laser as a source of excitation. The time-resolved photoluminescence decay spectra with fitting curves and their corresponding parameters for the 2D BCNO nanophosphor are shown in Fig. 4d. The PL decay was recorded for a $580 \mathrm{~nm}$ emission at $375 \mathrm{~nm}$ excitation wavelength. For the BCNO nanophosphor the decay time is in the range of nanoseconds. Since excitation lifetime is a size dependent parameter, the smaller the size of the nanophosphor, the shorter would be the excitation lifetime and the higher would be its recombination rate. The lifetime data of the $2 \mathrm{D}$ BCNO nanophosphor transitions are very well fitted to a double-exponential function as described by the equation

$$
I(t)=A_{1} \exp \left(-t / \tau_{1}\right)+A_{2} \exp \left(-t / \tau_{2}\right)
$$

where $\tau_{1}$ and $\tau_{2}$ are the decay lifetimes of the luminescence, and $A_{1}$ and $A_{2}$ are the weighting parameters. The observed lifetimes for the BCNO nanophosphor are $\tau_{1} \sim 0.311 \mathrm{~ns}$ and $\tau_{2} \sim 1.412 \mathrm{~ns}$. In the 2D BCNO nanophosphor, there are many defects such as $\mathrm{N}$ vacancy, substitutional defects ( $\mathrm{CB}$ or $\mathrm{CN}$ ) and $\mathrm{B} / \mathrm{O}$-related defects in the crystalline parts as well as near crystalline sites in the amorphous parts and these defects would generate different defect levels and affect the fluorescent lifetime. The obtained lifetime results showed second order exponential decay, which is consistent with an earlier reported paper on the BCNO nanophosphor. ${ }^{47}$ However, the value of the decay components depends upon the quality, composition, intrinsic and extrinsic parameters of the material. For doubleexponential decay, the average lifetime $\left(\tau_{\mathrm{av}}\right)$ is determined by the following equation.

$$
\tau_{\mathrm{av}}=\left(A_{1} \tau_{1}^{2}+A_{2} \tau_{2}^{2}\right) /\left(A_{1} \tau_{1}+A_{2} \tau_{2}\right)
$$

The average lifetime for the BCNO nanophosphor is calculated to be $\tau_{\mathrm{av}} \sim 0.4 \mathrm{~ns}$.

\subsection{Fabrication and characterization of CoB based WLEDs}

For the fabrication of WLEDs, the as synthesized yellow 2D BCNO nanophosphor was dispersed in epoxy resin with a curing agent. A solvent exchange process was developed to disperse the 2D BCNO nanophosphor in an epoxy resin with an appropriate amount. We tried several polymers such as silicon, poly(methyl methacrylate) (PMMA), epoxy, and polycarbonate (PC); we then chose epoxy resin because of its higher refractive index (1.5), so as to decrease total internal reflection. Initially, $20 \mathrm{mg}$ of $2 \mathrm{D}$ BCNO nanophosphor was dispersed in an epoxy solution (a mixture of epoxy resin and a curing agent which acts as a hardener). A spray set-up was used to coat the solution of 2D BCNO nanophosphor on CoB based blue LEDs. The ratio of epoxy resin and hardener was $100: 12.5$ by volume. The above mixture was uniformly mixed to make it homogeneous. After that, it was carefully spray-coated on CoB based blue LEDs arrays (LED FLOOD LIGHT Outdoor display light; $10 \mathrm{~W}$ power, $120^{\circ}$ beaming angle, DC $12 \mathrm{~V}^{\prime}$, InGaN based ROHS, a ISO 9001 registered company, made in China). Afterwards, it was left without further treatment to dry at room temperature for few hours before testing. The detailed fabrication protocol of CoB based WLEDs from the as synthesized 2D BCNO nanophosphor integrated to $\mathrm{CoB}$ based blue LEDs is shown in Scheme S2 (see ESI $\dagger$ ).

Prior to the fabrication of the 2D BCNO nanophosphor integrated to CoB based blue LEDs, we performed the PL experiment at different temperatures to explore the PL stability (thermal quenching effect), as shown in Fig. S13 (see ESI $\dagger$ ). The obtained results clearly demonstrate that this 2D BCNO nanophosphor is highly stable at all the performed temperatures (room temperature, 50, 100, 150, 200 and $250{ }^{\circ} \mathrm{C}$ ). The experiment was especially performed up to $250{ }^{\circ} \mathrm{C}$ because it is likely that the heat dissipation from the CoB based blue LEDs arrays may generate a temperature up to $200{ }^{\circ} \mathrm{C}$.

Fig. 5a and b shows the uncoated $\mathrm{CoB}$ based blue LEDs arrays and the 2D BCNO nanophosphor coated CoB based blue LEDs arrays, respectively. To examine the homogeneity of nanophosphor coating on the blue CoB based LED, we performed PL mapping of the nanophosphor coated area. Fig. 5c shows uniform distribution of nanophosphor throughout the surface of the chip and the inset shows a clear view of uniform coating 

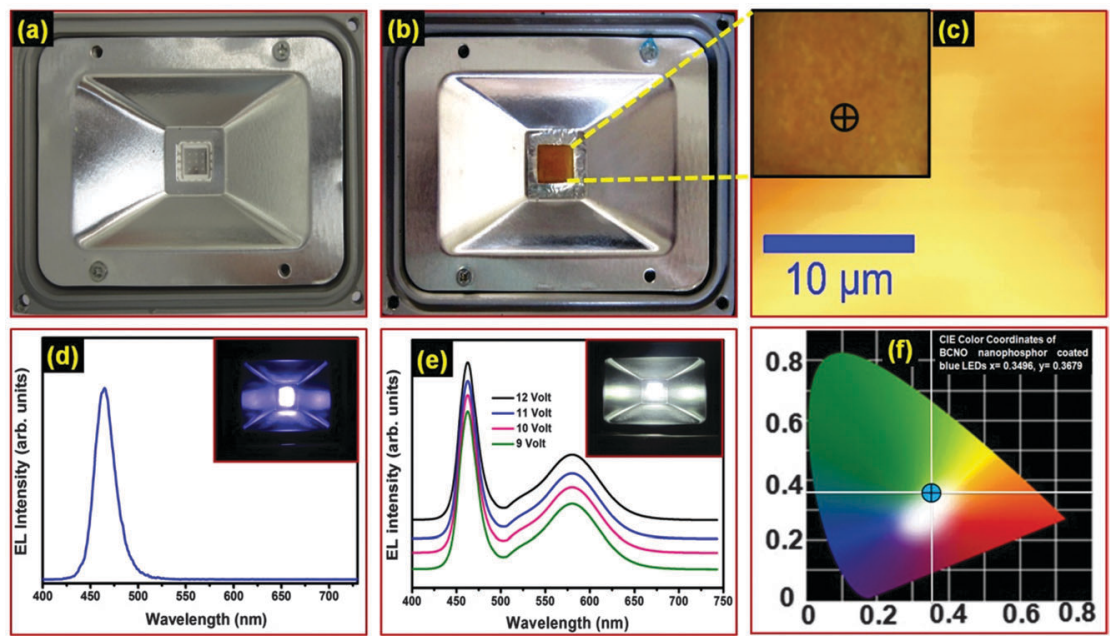

Fig. 5 (a) Uncoated CoB based blue LEDs arrays. (b) 2D BCNO nanophosphor coated CoB based blue LEDs arrays. (c) PL mapping of the 2D BCNO nanophosphor coated blue COB based blue LEDs; inset shows a clear view of uniform coating and the black cross mark shows the selected area where PL mapping was performed. (d) EL emission spectrum of a commercial CoB based blue LEDs without phosphor coating at 12 Volt DC; inset shows the optical image of a CoB based blue LEDs in the glowing mode. (e) White light EL emission spectrum of a fabricated nanophosphor coated blue CoB based LEDs at different voltages $(9,10,11$ and 12 Volt) DC power supplies; inset shows the optical image of the 2D BCNO yellow nanophosphor coated CoB based blue LEDs in the glowing mode. (f) CIE color co-ordinates of the 2D BCNO yellow nanophosphor coated CoB based blue LEDs.

and the black cross mark indicates the selected area where PL mapping was performed. The confocal microscope during the process of PL mapping is shown in Fig. S14 (see ESI $\dagger$ ). Fig. 5d reveals the electroluminescence (EL) emission spectrum of a commercial СoB based blue LEDs glowing on a 12 Volt DC power supply. The EL emission spectrum of the blue LED significantly overlaps with excitation spectra of the nanophosphor, as shown in Fig. 4a, which implies its suitability for pumping the yellow nanophosphor. Fig. 5e presents the EL spectrum of commercial CoB based blue LEDs coated with the as synthesized 2D BCNO nanophosphor glowing at different $(9,10,11,12)$ Volt DC power supplies. It reveals the blue to white light conversion with high efficiency through the yellow BCNO nanophosphor at 12 Volt power supply and efficiency decreases when it goes down to lower voltage. The insets of Fig. $5 \mathrm{~d}$ and e show the optical images of $\mathrm{CoB}$ based blue LEDs before and after 2D BCNO nanophosphor coating, respectively, in the glowing mode. The electroluminescence measurement instrument, along with yellow nanophosphor coated CoB based LEDs, is shown in Fig. S15 (see ESI $\dagger$ ). The color temperature and CIE color co-ordinates measurement instrument is shown in Fig. S16 (see ESI†). The CIE color co-ordinates of the BCNO yellow nanophosphor coated $\mathrm{CoB}$ based blue LED is shown in Fig. 5f. It is quite evident from this figure that emission of the BCNO yellow nanophosphor coated $\mathrm{CoB}$ lies considerably in the white region, having color co-ordinates $x=0.3496$ and $y=0.3679$. Thus, the obtained excellent results (color rendering index $\sim 92$, color temperature $\sim 4899 \mathrm{~K}$, luminous efficiency $\sim 83.4 \mathrm{~lm} \mathrm{~W}^{-1}$, and brightness $834 \mathrm{Cd} \mathrm{m}^{-2}$ ) of this $2 \mathrm{D}$ emerging BCNO nanophosphor show its potential applications in next generation rare-earth free $2 \mathrm{D}$ lightweight material based WLED devices (the brightness of the device 2D BCNO yellow nanophosphor coated CoB based blue LEDs was also tested for different time intervals to evaluate the feasibility of the device, as shown in Table S2 (see ESI $\dagger$ ), with an external quantum efficiency of the 2D BCNO nanophosphor coated $\mathrm{CoB}$ based blue LEDs arrays $\sim 73 \%$ ).

\section{Conclusions}

In summary, we have successfully synthesized a lightweight, rareearth free 2D BCNO (boron carbon oxynitride) nanophosphor by a facile customized auto-combustion method, which can be easily scaled up in large quantity at an economic cost. This 2D BCNO nanophosphor exhibits a broad highly intense yellow emission peak centered at $580 \mathrm{~nm}$ (using a $470 \mathrm{~nm}$ excitation wavelength) with a high quantum efficiency $\sim 89 \%$. The $2 \mathrm{D}$ BCNO nanophosphor coated on a CoB based blue LEDs device demonstrated better color coordinates $(x=0.3496, y=0.3679)$, lower color temperature $(4899 \mathrm{~K})$, better color rendering index ( $\sim 92)$, higher luminous efficiency $\left(\sim 83.4 \mathrm{~lm} \mathrm{~W}^{-1}\right)$, and higher brightness $\left(834 \mathrm{Cd} \mathrm{m}^{-2}\right)$. Finally, we envision that this novel rare-earth free 2D lightweight BCNO nanophosphor will enable new key successes to build WLED devices that can replace existing phosphors which can be potentially utilized in next generation WLEDs.

\section{Acknowledgements}

The authors wish to thank Director, N.P.L., New Delhi, for his keen interest in the work. The authors are thankful to Prof. O. N. Srivastava (Banaras Hindu University, Varanasi) for his encouragement. Mr Pawan Kumar gratefully acknowledges University Grant Commission (UGC), Govt. of India, for financial assistance under RGNF Research Fellowship, Award No. F1-17.1/2011-12/RGNF-SCPUN-12604/(SA-III/Website). The authors are grateful to the CSIR TAPSUN program providing PL instrument facility. 


\section{References}

1 A. K. Geim and K. S. Novoselov, Nat. Mater., 2007, 6, 183-191.

2 K. S. Novoselov, A. K. Geim, S. V. Morozov, D. Jiang, Y. Zhang, S. V. Dubonos, I. V. Grigorieva and A. A. Firsov, Science, 2004, 306, 666-669.

3 G. Gao, W. Gao, E. Cannuccia, J. Taha-Tijerina, L. Balicas, A. Mathkar, T. N. Narayanan, Z. Liu, B. K. Gupta, J. Peng, Y. Yin, A. Rubio and P. M. Ajayan, Nano Lett., 2012, 12, 3518-3525.

4 A. A. Balandin, Nat. Mater., 2011, 10, 569-581.

5 H. C. Neto, F. Guinea, N. M. R. Peres, K. S. Novoselov and A. K. Geim, Rev. Mod. Phys., 2009, 81, 109-162.

6 Y. Kubota, K. Watanabe, O. Tsuda and T. Taniguchi, Science, 2007, 317, 932-934.

7 K. Watanabe, T. Taniguchi, T. Niiyama, K. Miya and M. Taniguchi, Nat. Photonics, 2009, 3, 591-594.

8 L. Song, Z. Liu, A. L. M. Reddy, N. T. Narayanan, J. TahaTijerina, J. Peng, G. Gao, J. Lou, R. Vajtai and P. M. Ajayan, Adv. Mater., 2012, 24, 4878-4895.

9 H. B. Zeng, C. Y. Zhi, Z. H. Zhang, X. L. Wei, X. B. Wang, W. L. Guo, Y. Bando and D. Golberg, Nano Lett., 2010, 10, 5049-5055.

10 L. Song, L. J. Ci, H. Lu, P. B. Sorokin, C. H. Jin, J. Ni, A. G. Kvashnin, D. G. Kvashnin, J. Lou, B. I. Yakobson and P. M. Ajayan, Nano Lett., 2010, 10, 3209-3215.

11 W. Q. Han, H. G. Yu, C. Zhi, J. Wang, Z. Liu, T. Sekiguchi and Y. Bando, Nano Lett., 2008, 8, 491-494.

12 M. G. Silly, P. Jaffrennou, J. Barjon, J. S. Lauret, F. Ducastelle, A. Loiseau, E. Obraztsova, B. Attal-Tretout and E. Rosencher, Phys. Rev. B: Condens. Matter Mater. Phys., 2007, 75, 085205.

13 D. Portehault, C. Giordano, C. Gervais, I. Senkovska, S. Kaskel, C. Sanchez and M. Antonietti, Adv. Funct. Mater., 2010, 20, 1827-1833.

14 K. Raidongia, A. Nag, K. P. S. S. Hembram, U. V. Waghmare, R. Datta and C. N. R. Rao, Chem. - Eur. J., 2010, 16, 149.

15 L. W. Yin, Y. Bando, D. Golberg, A. Gloter, M. S. Li, X. L. Yuan and T. Sekiguchi, J. Am. Chem. Soc., 2005, 127, 16354-16355.

16 L. Liao, K. H. Liu, W. L. Wang, X. D. Bai, E. G. Wang, Y. L. Liu, J. C. Li and C. Liu, J. Am. Chem. Soc., 2007, 129, 9562-9563.

17 J. Yu and E. G. Wang, Appl. Phys. Lett., 1999, 74, 2948.

18 S. Y. Wang, L. P. Zhang, Z. H. Xia, A. Roy, D. W. Chang, J. B. Baek and L. M. Dai, Angew. Chem., Int. Ed., 2012, 51, 4209-4212.

19 H. Konno, T. Ito, M. Ushiro, K. Fushimi and K. Azumi, J. Power Sources, 2010, 195, 1739-1746.

20 X. Liu, S. Ye, Y. B. Qiao, G. P. Dong, Q. Zhang and J. R. Qiu, Chem. Commun., 2009, 4073-4075.

21 W. W. Lei, D. Portehault, R. Dimova and M. Antonietti, J. Am. Chem. Soc., 2011, 133, 7121-7127.

22 J. Dwivedi, P. Kumar, A. Kumar, X. X. Sudama, V. N. Singh, B. P. Singh, S. K. Dhawan, V. Shanker and B. K. Gupta, RSC Adv., 2014, 4, 54936-54947.

23 Y. Zhang, C. Xie, H. Su, J. Liu, S. Pickering, Y. Wang, W. W. Yu, J. Wang, Y. Wang, J. Hahm, N. Dellas, S. E. Mohney and J. Xu, Nano Lett., 2011, 11, 329-332.
24 H. P. T. Nguyen, S. Zhang, A. T. Connie, M. G. Kibria, Q. Wang, I. Shih and M. Zetian, Nano Lett., 2013, 13, 5437-5442.

25 Z. Yang, J. Xu, P. Wang, X. Zhuang, A. Pan and L. Tong, Nano Lett., 2011, 11, 5085-5089.

26 W. N. Wang, Y. Kaihatsu, F. Iskandar and K. Okuyama, Mater. Res. Bull., 2009, 22, 2099-2102.

27 Y. kaihatsu, W. N. Wang, K. Okuyama, T. Ogi and K. Okuyama, J. Electrochem. Soc., 2010, 157, J329-J333.

28 T. Ogi, F. Iskandar and A. B. D. Nandiyanto, J. Chem. Eng. Jpn., 2012, 45, 995-1000.

29 T. Ogi, H. Iwasaki, A. B. D. Nandiyanto, F. Iskandar, W. N. Wang and K. Okuyama, J. Mater. Chem. C, 2014, 2, 4297-4303.

30 T. Ogi, Y. Kaihatsu, F. Iskandar, W. Wang and K. Okuyama, Adv. Mater., 2008, 20, 3235-3238.

31 Y. Kaihatsu, F. Iskander, H. Vidyandari, W. Wang and K. Okuyama, Electrochem. Solid-State Lett., 2009, 12, J33-J36.

32 A. Rubio, J. L. Corkill and M. L. Cohen, Phys. Rev. B: Condens. Matter Mater. Phys., 1994, 49, 5081.

33 M. S. C. Mazzoni, R. W. Nunes, S. Azevedo and H. Chacham, Phys. Rev. B: Condens. Matter Mater. Phys., 2006, 73, 073108.

34 M. O. Watanabe, S. Itoh, T. Sasaki and K. Mizushima, Phys. Rev. Lett., 1996, 77, 187.

35 X. D. Bai, E. G. Wang, J. Yu and H. Yang, Appl. Phys. Lett., 2000, 77, 67.

36 W. N. Wang, T. Ogi, Y. Kaihatsu, F. Iskandarc and K. Okuyama, J. Mater. Chem., 2011, 21, 5183-5189.

37 L. Qin, J. Yu, S. Kuang, C. Xiao and X. Bai, Nanoscale, 2012, 4, 120-123.

38 G. Kedawat, S. Srivastava, V. K. Jain, P. Kumar, V. Kataria, Y. Agrawal, B. K. Gupta and Y. K. Vijay, ACS Appl. Mater. Interfaces, 2013, 5, 4872-4877.

39 B. K. Gupta, D. Haranath, S. Saini, V. N. Singh and V. Shanker, Nanotechnology, 2010, 21, 055607.

40 K. Mishra, S. K. Singh, A. K. Singh, M. Rai, B. K. Gupta and S. B. Rai, Inorg. Chem., 2014, 53, 9561-9569.

41 G. R. Bhimanapati, D. Kozuch and J. A. Robinson, Nanoscale, 2014, 6, 11671-11675.

42 P. Thanikaivelan, T. N. Narayanan, L. Song, W. Gao, T. Hayashi, A. L. M. Reddy, A. Saha, V. Shanker, M. Endo, A. A. Marti and P. M. Ajayan, Nano Lett., 2011, 11, 5227-5233.

43 M. Hubacek, T. Sato and T. Ishii, J. Solid State Chem., 1994, 109, 384-390.

44 V. Brozek and M. Hubacek, J. Solid State Chem., 1992, 100, 120-129.

45 M. A. Mannan, M. Nagano, K. Shigezumi, T. Kida, N. Hirao and Y. Baba, Am. J. Appl. Sci., 2008, 5, 736-741.

46 C. C. Tang, Y. Bando, C. Y. Zhi and D. Golberg, Chem. Commun., 2007, 4599-4601.

47 X. Zhang, Z. Lu, J. Lin, L. Li, Y. Fan, L. Hu, X. Xu, F. Meng, J. Zhao and C. Tang, Mater. Lett., 2013, 94, 72-75.

48 X. Zhang, L. Li, Z. Lu, J. Lin, X. Xu, Y. Ma, X. Yang, F. Meng, J. Zhao and C. Tang, J. Am. Ceram. Soc., 2014, 97, 246-250.

49 L. Museur, E. Feldbach and A. Kanaev, Phys. Rev. B, 2008, 78, 1552049. 